\title{
Fluorescent Mitoxantrone Hydrochloride Nanoparticles Inhibit the Malignant Behavior of Giant Cell Tumor of Bone via miR- 125b/PTH1R Axis
}

\author{
Baohui Su, ${ }^{1}$ Yanguang Yuan, ${ }^{2}$ Junshan Zhang, ${ }^{1}$ Yuezhong $\mathrm{Li}^{1}{ }^{1}$ and Qihui Zhang $\mathbb{D}^{3}$ \\ ${ }^{1}$ Department of Spinal Surgery, Weifang People's Hospital, Weifang, Shandong 261041, China \\ ${ }^{2}$ Department of Orthopaedics, Anqiu People's Hospital, Weifang, Shandong 262100, China \\ ${ }^{3}$ Department of Nursing, Anqiu People's Hospital, Weifang, Shandong 262100, China
}

Correspondence should be addressed to Qihui Zhang; 2580332003@qq.com

Received 19 May 2020; Accepted 6 July 2020; Published 1 August 2020

Guest Editor: Dongyu Li

Copyright (c) 2020 Baohui Su et al. This is an open access article distributed under the Creative Commons Attribution License, which permits unrestricted use, distribution, and reproduction in any medium, provided the original work is properly cited.

\begin{abstract}
Objective. To explore the therapeutic effects and mechanism of fluorescent mitoxantrone hydrochloride nanoparticles on giant cell tumor of bone. Methods. The adsorption capacity of nanoparticles to hydroxyapatite (HA), cell adsorption capacity, encapsulation rate, particle size, and potential of the nanoparticles were determined by HPLC and Zetasizer Nano ZS nanomicelle potentiometer. MTT assay was used to determine the toxicity of nanoparticles to cells. The fluorescent intensity of the nanoparticles and their location in the cells were observed under a fluorescence microscope. RT-qPCR and Western blotting were then used to measure the expression levels of miRNA, mRNA, and proteins in cells. Transwell and Annexin V-FITC/PI staining tests were used to study the cell invasion and apoptotic rate, respectively. The dual-luciferase reporter gene experiment was then carried out to verify the binding relationship between miR-125b and its predicted target. Results. ALN-FOL-MTO-NLC nanoparticles showed a stronger adsorption capacity for HA and stronger toxicity to GCTB28 cells. Compared to normal tissues, the expression level of miR-125b in giant bone tumor tissue and cells was significantly downregulated, and the expression level of miR-125b was upregulated to some extent after treatment. Overexpression of miR-125b or treatment of ALN-FOL-MTO-NLC nanoparticles can inhibit the malignant behavior of GCTB28 cells, whereas the inhibition of the expression of miR-125b can promote the malignant behavior of GCTB28 cells. The result showed that parathyroid hormone receptor 1 (PTH1R) was a downstream target gene for miR-125b. Rescue experiment showed that the treatment of GCTB28 with ALN-FOL-MTO-NLC nanoparticles while inhibiting miR-125b expression can reduce the inhibitory effect of miR-125b on the malignant behavior of GCTB28 cells, whereas upregulating the expression levels of miR-125b and PTH1R in GCTB28 cells had no significant effect on the malignant behavior of GCTB28 cells. Conclusion. ALN-FOL-MTO-NLC nanoparticles have a certain inhibitory effect on the malignant behavior of giant cell tumor of bone through the miR-125b/PTH1R molecular axis.
\end{abstract}

\section{Introduction}

Giant cell tumor of bone (GCT) is a bone tumor with high invasiveness and osteolytic nature [1] with potential malignancy [2]. GCT is mainly composed of three kinds of cells, including bone cell-like multinucleated giant cells, spindlelike stromal cells, and monocytes [3]. At present, the preferred treatment for GCT is local treatment with assisted surgery, but this treatment has a higher postoperative recurrence rate [4]; therefore, it is important to find a new way for GCT treatment.
Considering the structural characteristics of human tissues, nanoparticles have good advantages in size, can interact with the biological components of the human body, and regulate various biological behaviors of the human body [5]. Nanomaterial-based drug delivery systems are smaller in size, giving them quantum size effects, interfacial effects, and macrochain tunneling effects. Therefore, the nanodrug showed strong permeability, greater solubility, better adsorption, and so on in biology. Due to the small size and large surface area of nanoparticles, nanodrugs are easy to pass 
through blood vessels in vivo and are not easy to cause damage to the inner wall of blood vessels. In addition, the nanoparticles have the advantages of high surface activity, many active centers, and good catalytic efficiency, so nanodrugs can be prepared into sustained-release drugs, change the half-life of drugs in vivo, and extend the action time of drugs. Based on the above advantages, the drug delivery system based on nanomaterials can have better targeting, so that the nanodrug-carrying system can pass through the biological barrier and reach the lesion area [6]. Not surprisingly, a large number of studies have shown that drug delivery systems based on nanomaterials can be used to treat a variety of orthopedic diseases that are difficult to treat with conventional clinical therapies, such as arthritis, osteoporosis, bone cancer, and related bone diseases [7-11]. Although nanoparticles have certain advantages in the treatment of bone diseases, there are still several problems, such as the low drug loading of bonetargeted macromolecular carriers and its easy excretion through the kidney.

Mitoxantrone hydrochloride (Mitoxantro, MTO) is usually used as an anticancer drug in cancer treatment. Shi et al. took MTO as a model drug and developed a new kind of nanodrug-carrying system, and the results of in vivo and in vitro experiments confirmed that the nanodrug-carrying system has a good long-circulation effect and an increased ability to target bone tissue [12]. However, the above research only discussed the theoretical advantages of the new nanodrug-loading system and does not study whether the nanodrug-loading system can improve the therapeutic effect on bone tumors.

MicroRNA (miRNA, miR) is a group of endogenous noncoding RNAs with a length of 20 to 25 nucleotides. It can negatively regulate the expression of mRNA by inhibiting the translation of mRNA or reducing the stability of mRNA [13]. Studies have shown that miRNA can play an important role in many biological processes. Parathyroid hormonerelated protein (PTHrP) plays a pivotal role in the development of bones [14], and its expression in various cancer tissues and cells is also significantly upregulated [15]. PTH1R as a PTHrP receptor is also positively expressed in various cancers and cells [16]. In the previous research, PTH1R was found to be the target gene of miR-125b through the prediction of biological gene database. Therefore, in this article, we will discuss the treatment effects of GCT with the nanodrugloaded particles at the cellular level and verify the mechanism of action of miR-125b/PTH1R in GCT.

\section{Materials and Methods}

\subsection{Experimental Materials}

2.1.1. Tissues and Cells. Bone giant cell tumor tissues and corresponding paracancerous tissues were collected from patients who were diagnosed and operated in our hospital from October 2010 to October 2019. This study was approved by the ethics committee of our hospital. Both patients and their families knew the purpose and significance of this study and signed the informed consent. MG-63 cells and hFOB1.9 cells were purchased from Tongpai (Shanghai) Biotechnology Co., Ltd. GCTB28 cells were purchased from China Center for Type Culture Collection.

2.1.2. Experimental Reagents. Phospholipid, glyceryl trilaurate, cholesterol, mitoxantrone hydrochloride, sodium lauryl sulfate, methanol, methylene chloride, FOL-S100, ALN-S100, hydroxyphosphorus lime, coumarin-6, dimethyl sulfoxide, and polyoxyethylene castor oil were all purchased from Shanghai Houcheng Fine Chemical Co., Ltd. RPMI 1640 medium, Annexin V-FITC/PI kit, and QuikChange sitedirected mutation kit were purchased from Shanghai Kemin Biotechnology Co., Ltd. The BCA kit and TRIZOL kit were purchased from Beijing Kairuiji Biotechnology Co., Ltd. The MTT test kit was purchased from Shanghai Yiyan Biotechnology Co., Ltd. The RT kit (reverse transcription kit) was purchased from Shanghai Zeye Biotechnology Co., Ltd. RIPA lysate was purchased from Shanghai Yuanye Biotechnology Co., Ltd., and the dual-luciferase reporter gene kit was purchased from Beijing Baierdi Biotechnology Co., Ltd.

2.2. Preparation of Mitoxantrone Hydrochloride Nanoparticles. $250 \mathrm{mg}$ of a phospholipid, $25 \mathrm{mg}$ of glyceryl trilaurate, $25 \mathrm{mg}$ of cholesterol, $10 \mathrm{mg}$ of mitoxantrone hydrochloride, and $10 \mathrm{mg}$ of sodium lauryl sulfate were weighed and added to a $250 \mathrm{~mL}$ eggplant-shaped bottle, and $10 \mathrm{~mL}$ of methanol-dichloromethane (volume ratio $1: 1$ ) mixture was then added. The solution was then distilled at $37^{\circ} \mathrm{C}$ under reduced pressure and vacuum dried overnight. After that, $10 \mathrm{~mL}$ of $2 \%$ polyoxyethylene castor oil aqueous solution was added and hydrated at $37^{\circ} \mathrm{C}$ for $30 \mathrm{~min}$. It was then immersed in an ice bath $(200 \mathrm{~W})$ for 300 times and squeezed through a $0.22 \mu \mathrm{m}$ filter to obtain toxantrone hydrochloride nanoparticles (MTO-NLCs).

To obtain the ALN-FOL-MTO-NLCs, $20 \mathrm{mg}$ of folic acid-polyoxyethylene monostearate (FOL-S100) and $100 \mathrm{mg}$ of alenic acid-polyoxyethylene monostearate (ALN-S100) were added to the eggplant-shaped flask before distillation under reduced pressure. The subsequent steps were the same as above, and the double-ligand modified nanoparticles (ALN-FOL-MTO-NLCs) were then obtained.

2.3. Measurement of Nanoparticle Encapsulation Rate, Particle Size, and Potential. $0.1 \mathrm{~mL}$ of nanoparticle was added with $0.4 \mathrm{~mL}$ of distilled water for dilution, which was then centrifuged at $8000 \mathrm{runs} / \mathrm{min}$ for $5 \mathrm{~min}$. The encapsulation rate was then calculated based on the drug content in supernatant and nanoparticle determined by HPLC. $0.1 \mathrm{~mL}$ of nanoparticle preparation was added in $3.9 \mathrm{~mL}$ of distilled water for dilution. Particle size and potential of nanoparticles were then determined with the Zetasizer Nano ZS nanomicelle potentiometer.

2.4. Identification of HA Adsorption Capacity of Nanoparticles. The nanoparticles were diluted with distilled water to make the drug content reach $0.1 \mathrm{mg} / \mathrm{mL}$. Two grams of hydroxyapatite (hydroxylapatite, HA) was then weighed and added into $50 \mathrm{~mL}$ of the diluted nanoparticle preparation and stirred at room temperature for $60 \mathrm{~min}$, which was then sampled every 15 minutes. After filtering the sample through 
a $0.45 \mu \mathrm{m}$ filter membrane, the drug content in the unabsorbed nanoparticles and the diluted nanoparticle preparation in the filtrate were determined by HPLC, and the adsorption ratio of the nanoparticles was then calculated.

2.5. Cellular Adsorption Capacity of Nanoparticles. Instead of mitoxantrone hydrochloride and sodium lauryl sulfate, coumarin-6 (C6) was used to prepare the C6-coated nanoparticles according to the method in Section 2.2. GCTB28 cells in the logarithmic growth phase were seeded in 96well plates at a density of $1 \times 10^{5}$ per well and incubated at $37^{\circ} \mathrm{C}$ for $24 \mathrm{~h}$. After discarding the culture solution, the nanoparticle preparation diluted with RPMI 16140 medium without folic acid was added, the cells were then incubated at different temperatures for $4 \mathrm{~h}$ and washed 3 times with PBS after the incubation. After lysing the cells, the BCA kit and HPLC were then used to determine the concentration of protein and C6 in the sample. The uptake index was calculated according to the formula: uptake $(\%)=$ intracellular C6 concentration/intracellular protein concentration $\times 100$.

2.6. Determination of Cytotoxicity of Nanoparticles. GCTB28 cells in the logarithmic growth phase were seeded in 96-well plates at a density of $5 \times 10^{3}$ per well. MTO-NLCs and ALNFOL-MTO-NLCs were diluted with folic acid-free PRMI 1640 medium and added to each well, and the cells were then incubated for $48 \mathrm{~h}$. After that, each well was rinsed 3 times with $37^{\circ} \mathrm{C}$ HBSS, and $20 \mu \mathrm{L}$ of MTT solution was then added to each well. After incubation for another $4 \mathrm{~h}, 150 \mu \mathrm{L}$ of DMSO was then added. A microplate reader was then used to measure the absorbance of each well at $570 \mathrm{~nm}$ after the solution was thoroughly mixed.

2.7. Cell Transfection. Cells in the logarithmic growth phase were taken and prepared into a cell suspension, seeded in 96-well plates at a density of $1 \times 10^{5}$ per well, and cultured at $37^{\circ} \mathrm{C}$ for $24 \mathrm{~h}$. The Liposome 2000 kit instructions were then strictly followed for cell transfection, and the transfection efficiency was determined with the RT-qPCR experiment.

2.8. $R T-q P C R$. The total RNA in the cells was extracted with the TRIzol kit, and then reverse transcription was performed using the reverse transcription kit according to the reaction system shown in Table 1 . The expression levels of miRNA and mRNA in cells were quantitatively measured based on the reaction system shown in Table 2. The U6 and GAPDH were used as internal references for miRNA and mRNA, respectively. The setting for PCR was as follows: 40 total cycles, $95^{\circ} \mathrm{C}$ for $10 \mathrm{~min}, 95^{\circ} \mathrm{C}$ for $30 \mathrm{~s}, 58^{\circ} \mathrm{C}$ for $30 \mathrm{~s}, 72^{\circ} \mathrm{C}$ for $10 \mathrm{~s}$, and $72^{\circ} \mathrm{C}$ for $10 \mathrm{~min}$.

2.9. MTT Experiment. Cells in the logarithmic growth phase of each group were pipetted into single cells in a serum-free medium for suspension culture. Cells were then seeded in 96-well plates at a density of $5 \times 10^{3}$ cells per well. After different treatments, $20 \mu \mathrm{L}$ of MTT solution dissolved in PBS was added. After incubating at $37^{\circ} \mathrm{C}$ for $4 \mathrm{~h}$, the culture medium in each well was discarded, and $150 \mu \mathrm{L}$ of DMSO
TABLE 1: Reverse transcription reaction system.

\begin{tabular}{lc}
\hline Material & Volume $(\mu \mathrm{L})$ \\
\hline RNA & 2 \\
oligoDT & 1 \\
Random primers & 1 \\
5X buffer & 4 \\
Mix & 1 \\
Deionized water & 12 \\
\hline
\end{tabular}

TABLE 2: PCR reaction system.

\begin{tabular}{lc}
\hline Material & Volume $(\mu \mathrm{L})$ \\
\hline SYBR Green Mix & 9 \\
cDNA & 2 \\
Primer & 1 \\
Deionized water & 8 \\
\hline
\end{tabular}

was added and thoroughly mixed. The absorbance of each well at $570 \mathrm{~nm}$ was then measured with a microplate reader.

2.10. Transwell Experiment. Cells in the logarithmic growth phase were seeded on the upper layer of the Transwell chamber coated with Matrigel gel, and the conventional medium was added to the lower layer of the Transwell chamber. After $24 \mathrm{~h}$, the cells were fixed with a mixture of acetic acid and formaldehyde for $15 \mathrm{~min}$, which was then rinsed three times with the PBS buffer. The cells that did not pass through the chamber were then gently wiped out with a cotton swab. Cells that passed through the chamber were then stained with crystal violet and washed three times with PBS buffer. To determine the cell invasiveness, the stained cells were then put under a microscope, and a number of field views were randomly selected to take pictures.

2.11. Western Blotting. Cells were lysed with RIPA lysate, and the proteins in the cells were separated using 10\% SDSPAGE, which was then transferred to PVDF membranes. The PVDF membranes were then blocked and subsequently incubated with primary antibody (PTH1R, $1: 1000)$ at $4^{\circ} \mathrm{C}$ overnight. After washing with PBS, the PVDF membranes were incubated with secondary antibody $(1: 5000)$ for $1 \mathrm{~h}$ at room temperature. Using GAPDH as an internal parameter, ImageJ was then used to determine the gray value of the band.

2.12. Annexin V-FITC/PI Double-Staining Experiment. After treating the cells of each group according to the experimental design, the cell culture was centrifuged at $3000 \mathrm{runs} / \mathrm{min}$ for $5 \mathrm{~min}$, and the supernatant was discarded. The pellet was then rinsed with PBS for 3 times. The collected cells were then seeded in a 96-well plate at a density of $1 \times 10^{5}$ per well and incubated for $12 \mathrm{~h}$. The Annexin V-FITC/PI doublestaining experiment was then carried out according to the instructions of Annexin V-FITC/PI Apoptosis Detection Kit. The apoptotic rates of each group of cells were then measured with flow cytometry. 


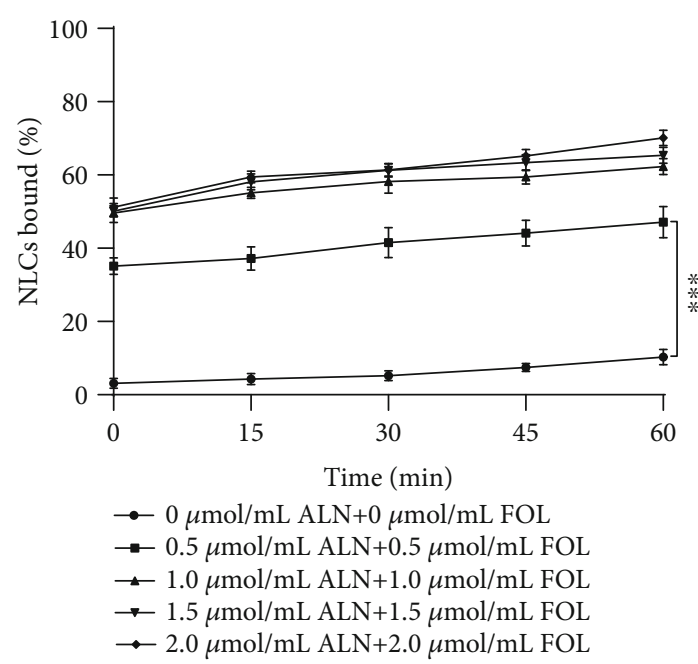

(a)

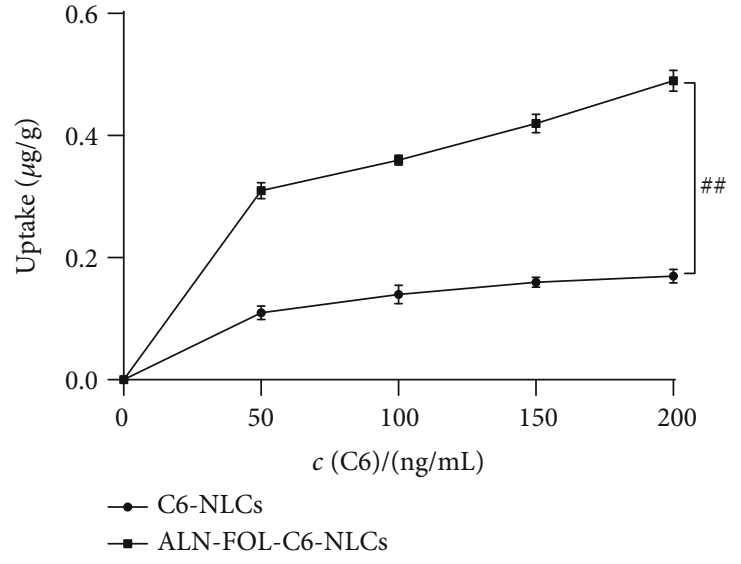

(b)

FIgure 1: Preparation and identification of nanoparticles. (a) The binding ability of MTO-loaded NLCs to HA. (b) Cell uptake of C6-NLCs and ALN-FOL-C6-NLCs $(\bar{x} \pm s, n=3) . * * * P<0.001$ compared with the $0 \mu \mathrm{mol} / \mathrm{L}$ ALN $+0 \mu \mathrm{mol} / \mathrm{L}$ FOL group; ${ }^{\# \#} P<0.01$ compared with the C6-NLCs group.

\subsection{Dual-Luciferase Reporter Gene Experiment. The PTH1R} $3^{\prime}$-UTR fragment was amplified and cloned into a vector to construct wild-type PTH1R (PTH1R-wt). And the mutant PTH1R (PTH1R-mut) was constructed using the QuikChange site-directed mutation kit. The liposome transfection kit was then used to transfect miR-NC or miR-125b mimics and PTH1R-wt or PTH1R-mut into GCTB28 cells. After incubation for $48 \mathrm{~h}$, the luciferase activity was determined using the dual-luciferase reporter gene system.

2.14. Localization of Drugs in Cells via Immunofluorescence Microscopy. GCTB28 cells in the logarithmic growth phase were inoculated in a special laser confocal Petri dish. After $24 \mathrm{~h}$ of incubation, ALN-FOL-MTO-NLC nanoparticles were added and further incubated for another $1 \mathrm{~h}$. After that, the medium was removed, and the cells were rinsed with PBS twice. An immunofluorescence microscope was then used to detect the fluorescence intensity and localization of ALNFOL-MTO-NLCs in the cells.

2.15. Statistical Analysis. GraphPad Prism 8.0 was used for drawing and statistical analysis. The $t$-test was used for the comparison between two groups, and the single-factor analysis of variance was used for the comparison between multiple groups. $P<0.05$ was used to indicate that the difference is statistically significant.

\section{Results}

3.1. Preparation and Identification of Mitoxantrone Hydrochloride Nanoparticles. Microscopic examination results showed that MTO-NLCs and ALN-FOL-MTO-NLCs were spherical and regular in shape. The test results showed that the encapsulation rate, particle size, and potential of MTO-NLCs were $99.37 \pm 0.26 \%, 50.62 \pm 3.34 \mathrm{~nm}$, and $-3.42 \pm 1.13 \mathrm{mV}$, respectively; the encapsulation rate, par- ticle size, and potential of ALN-FOL-MTO-NLCs were $99.87 \pm 0.17 \%, \quad 46.18 \pm 2.53 \mathrm{~nm}, \quad$ and $-17.11 \pm 2.24 \mathrm{mV}$, respectively. The HA adsorption test showed that compared with MTO-NLCs, the adsorption capacity of ALN-FOLMTO-NLCs on HA was significantly enhanced, and with the increase of the modification of ALN-S100 and FOLS100, the adsorption capacity of nanoparticles on HA also increased $(P<0.001)$, as shown in Figure $1(\mathrm{a})$. The results of cell uptake experiments showed that compared with C6NLCs, the uptake of ALC-FOL-C6-NLCs by GCTB28 cells was higher $(P<0.01)$, as shown in Figure $1(\mathrm{~b})$.

3.2. The Effects of Mitoxantrone Hydrochloride Nanoparticles on GCTB28 Cells. The cytotoxicity test results showed that ALN-FOL-MTO-NLCs in GCTB28 cells showed stronger cytotoxicity $(P<0.05)$ than MTO-NLCs, as shown in Figure 2(a). After the treatment of GCTB28 cells with ALN-FOR-MTO-NLC nanoparticles, the red fluorescence of the drug can be observed and mainly distributed in the cytoplasm, as shown in Figure 2(b). Based on the above results, we believe that ALN-FOL-MTO-NLCs have a better inhibitory effect on the malignant behavior of GCTB28 cells.

The results of RT-qPCR experiments showed that the expression of miR-125b in MG-63 cells and GCTB28 cells was significantly downregulated $(P<0.05)$, consistent with the results of Fei et al. [17]. And the expression level of miR-125b in hFOB1.19 cells was 4.82 times than that of GCTB28 cells, as shown in Figure 2(c). The expression of miR-125b in normal tissues was 5.16 times higher than that of cancer tissues, as shown in Figure 2(d). After GCTB28 cells were treated with nanoparticles, the expression levels of miR$125 \mathrm{~b}$ in the MTO-NLCs group and ALN-FOL-MTO-NLCs group were 3.67 and 4.89 times higher than that of the control group, respectively, as shown in Figure 2(e), which is consistent with our predictions; therefore, ALN-FOLMTO-NLCs were selected for the subsequent experiments. 


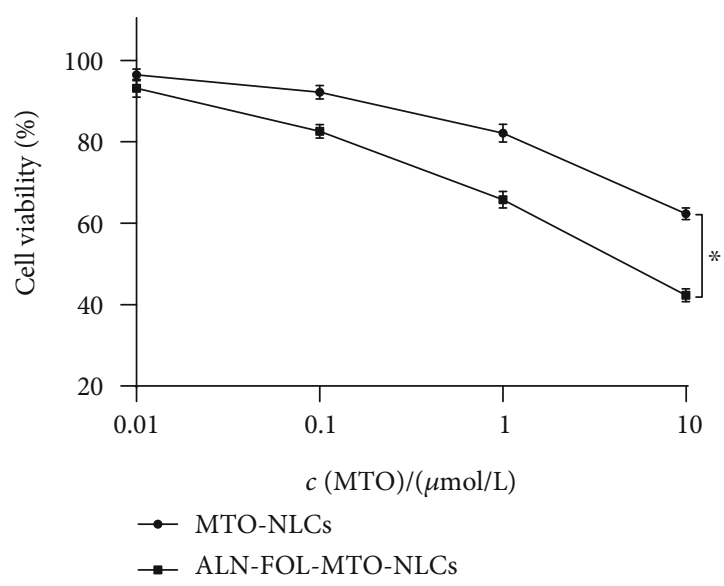

(a)

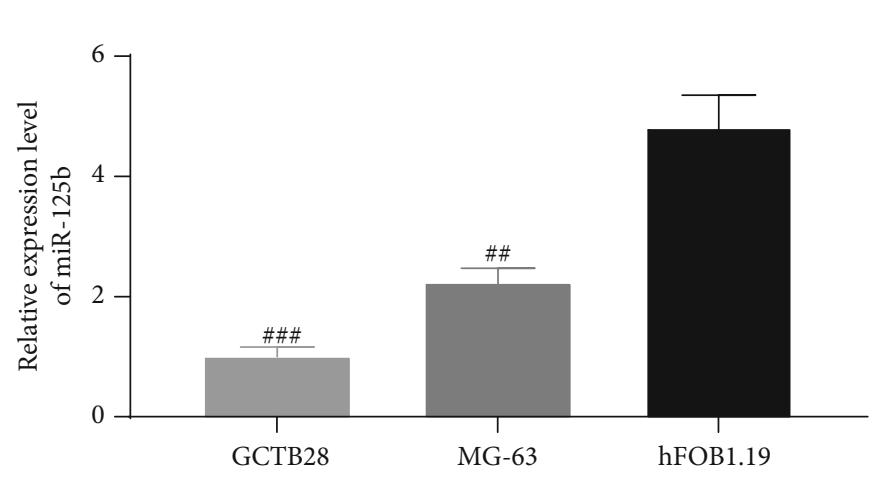

(c)

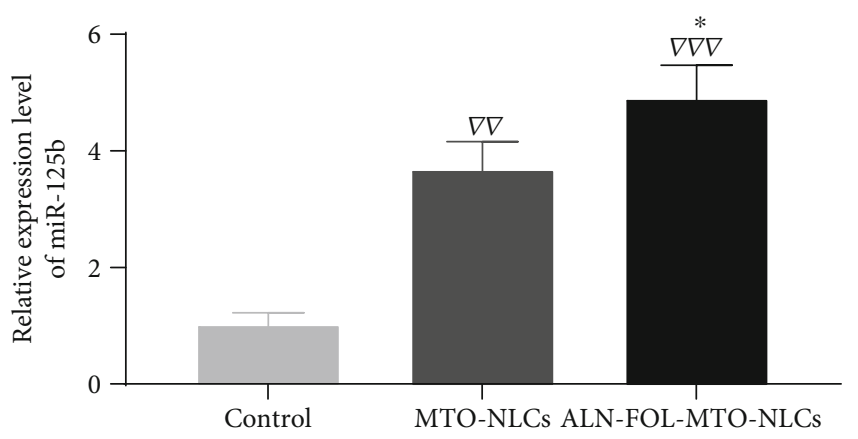

(e)

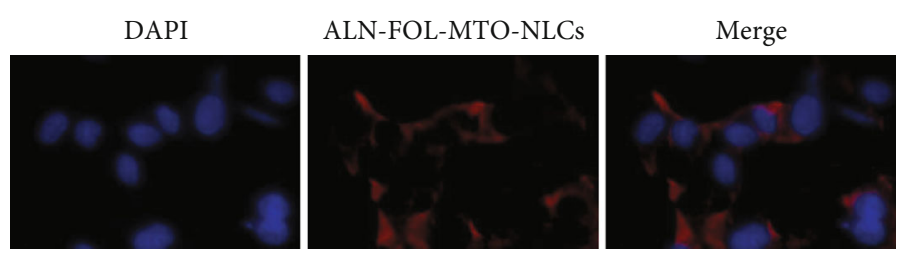

(b)

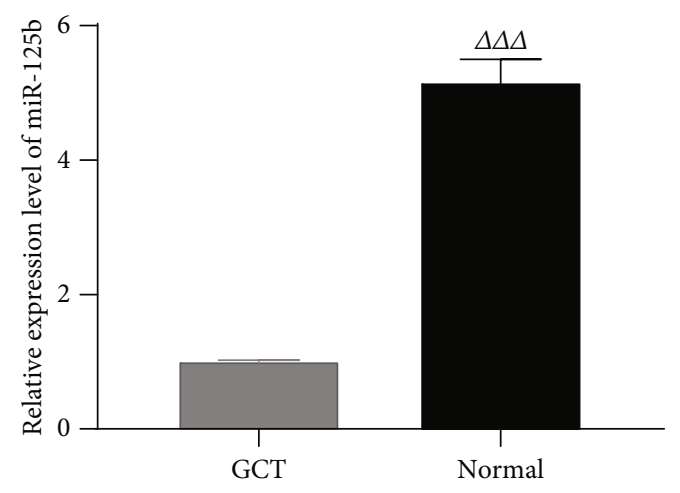

(d)

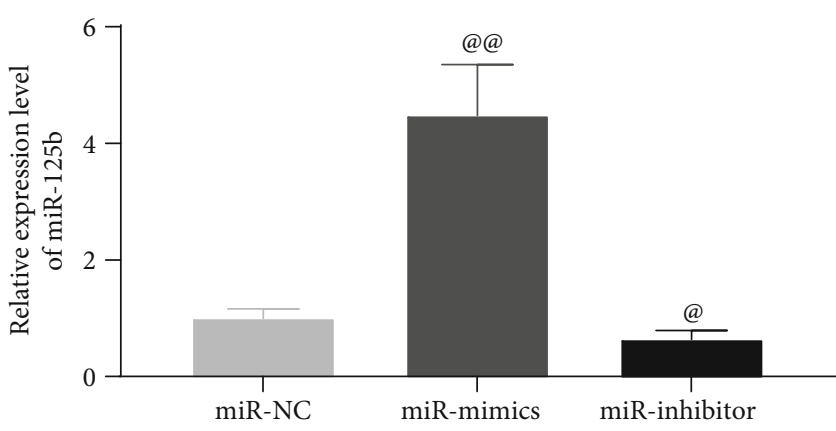

(f)

Figure 2: Continued. 


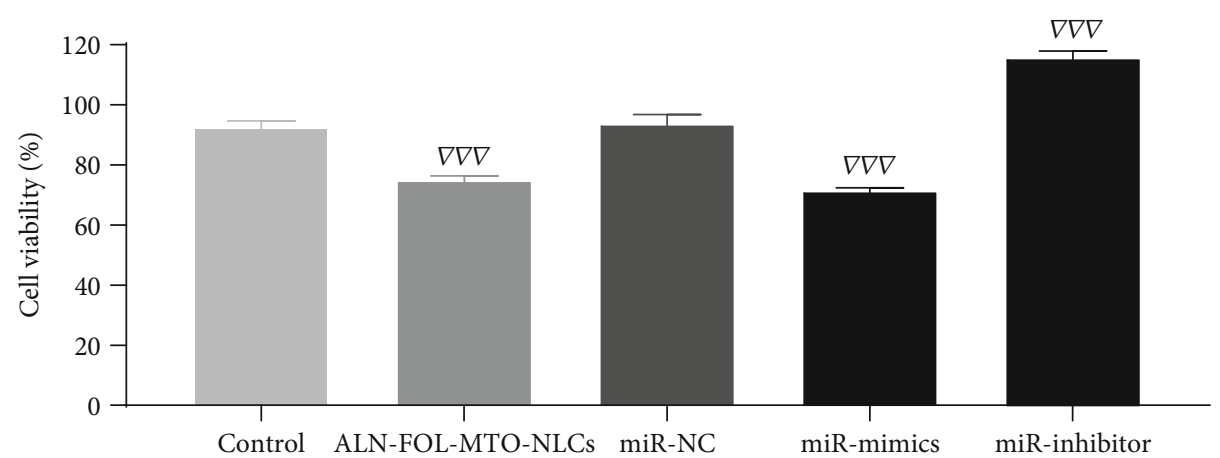

(g)
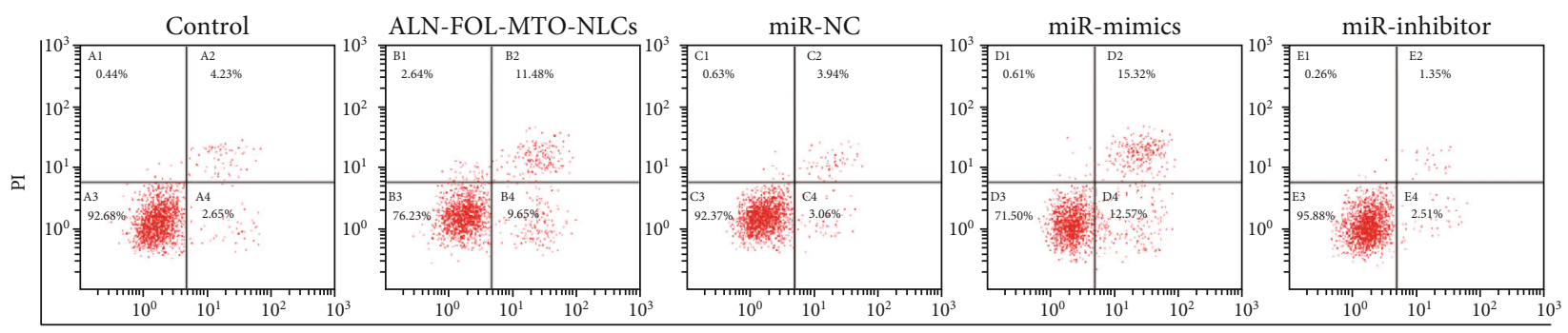

(h)

Control

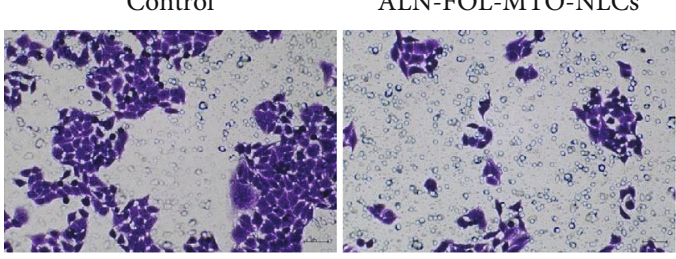

miR-NC

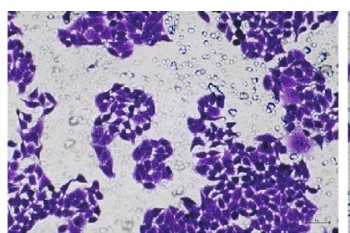

(i)

ANNEXIN V-FITC 


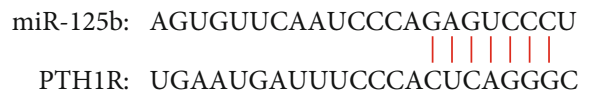

PTH1R: UGAAUGAUUUCCCACUCAGGGC

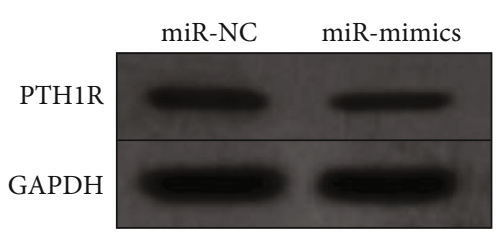

(c)

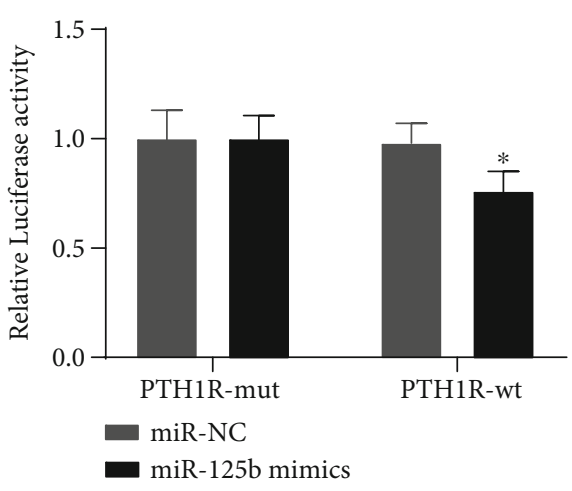

(b)

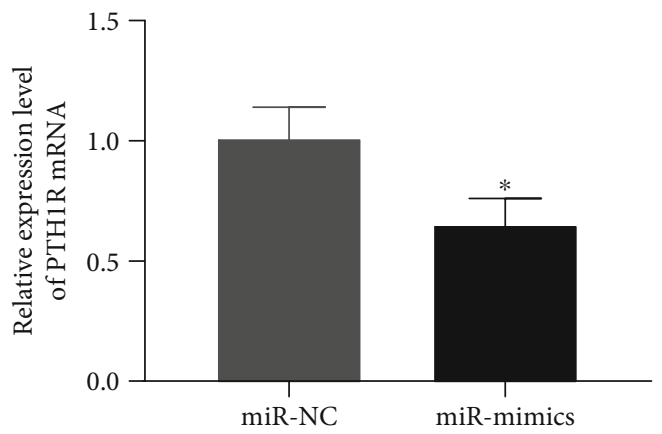

(d)

FIgure 3: Targeting relationship between miR-125b and PTH1R. (a) miR-125b and PTH1R-binding sequence. (b) Results of dual-luciferase reporter experiments. (c) Expression of PTH1R protein in GCTB28 cells. (d) The relative expression level of PTH1R in GCTB28 cells. $(\bar{x} \pm s$, $n=3) . * P<0.05$ compared with the miR-NC group.

it had no significant effect on the luciferase activity of PTH1R-mut, as shown in Figure 3(b). Thus, the above dual-luciferase reporter gene experiment verified the targeting relationship between PTH1R and miR-125b. Western blotting experiments and RT-PCR results also showed that overexpression of miR-125b significantly inhibited the expression of PTH1R protein and mRNA $(P<0.05)$, as shown in Figures 3(c) and 3(d).

3.4. ALN-FOL-MTO-NLCs Inhibit the Malignant Behavior of GCTB28 Cells through the miR-125b/PTH1R Molecular Axis. RT-qPCR results showed that the expression of PTH1R mRNA in GCTB28 cells was significantly increased after transfection with pc-PTH1R $(P<0.05)$, and the expression of PTH1R mRNA in GCTB28 cells was significantly decreased after transfection with si-PTH1R $(P<0.05)$, as shown in Figure 4(a). MTT results showed that compared with the control group, the proliferation activity of GCTB28 cells in the si-PTH1R group and ALN-FOL-MTO-NLCs group was significantly reduced $(P<0.05)$, whereas the proliferation activities of GCTB28 cells in the miR-inhibitor + ALN-FOL-MTO-NLCs group and miR-mimics + pcPTH1R group did not show significant changes, as shown in Figure 4(b). The Annexin V/PI double-staining results showed that compared with the control group, the apoptotic rates of GCTB28 cells in the si-PTH1R group and the ALNFOL-MTO-NLCs group increased significantly $(P<0.05)$, whereas there was no significant change in the apoptotic rates of GCTB28 cells in the miR-inhibitor + ALN-FOLMTO-NLCs group and miR-mimics + pc-PTH1R group, as shown in Figure 4(c) and Figure S3. Transwell experiment showed that compared with the control group, the number of invasive GCTB28 cell in the si-PTH1R group and the ALN-FOL-MTO-NLCs group was significantly reduced $(P<0.05)$, whereas the number of invasive GCTB28 cells in the miR-inhibitor + ALN-FOL-MTO-NLCs group and miR-mimics + pc-PTH1R group did not change significantly from the control group, as shown in Figure 4(d) and Figure S4.

\section{Discussion}

As one of the most common primary bone tumors, GCT is frequently occurred in young people and often happens around the knee joint. Although the malignant degree of GCT is low, it still has strong invasiveness and osteolysis, and the recurrence rate after surgical treatment is extremely high, which seriously affects the quality of patients' life. Therefore, finding new treatment strategies is essential to improve the quality of life for patients and reduce their recurrence rates after surgery. Studies have shown that nanoparticles have unparalleled advantages in the treatment of bone tumors. In this study, based on the research of Shi et al., MTO-NLC and ALN-FOL-MTO-NLC nanoparticles were prepared. Careful examination of the prepared nanoparticles revealed that both nanoparticles were spherical and regular 


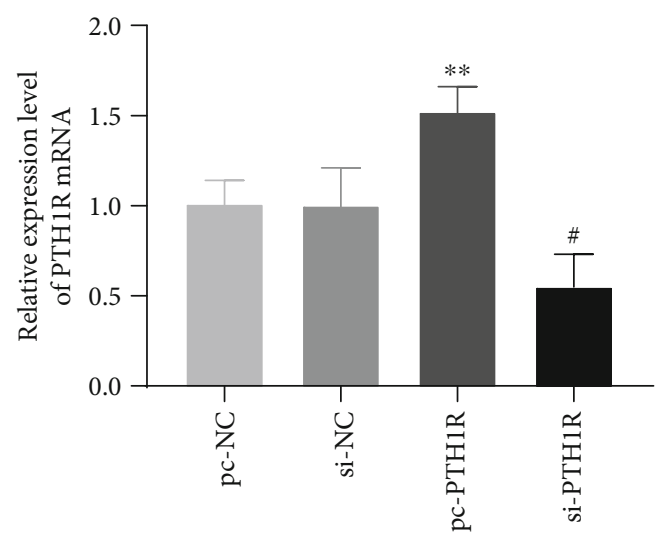

(a)

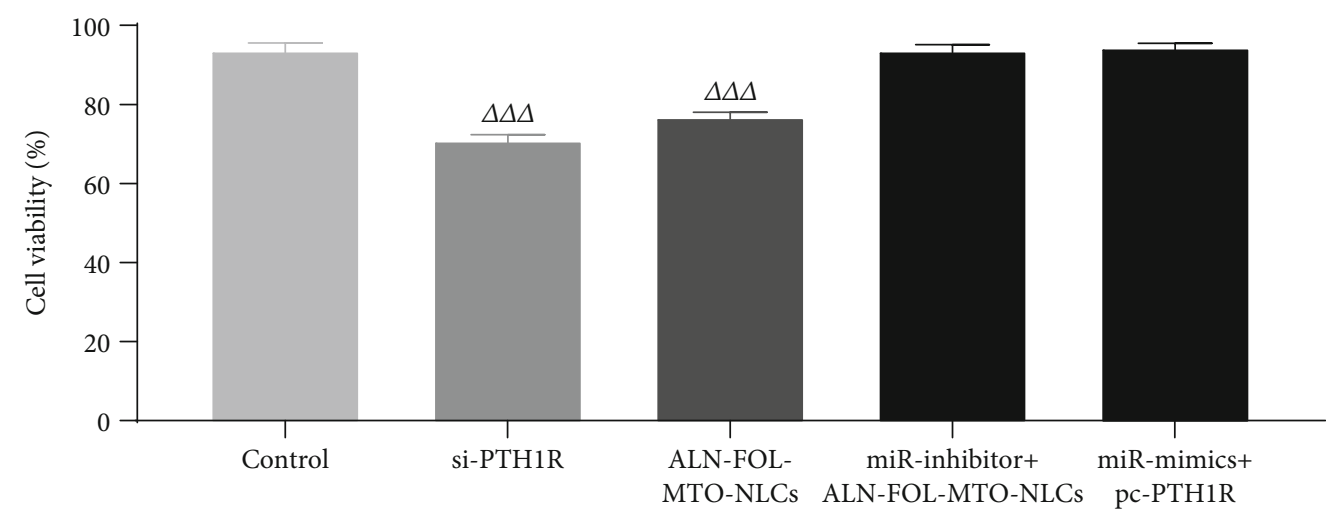

(b)

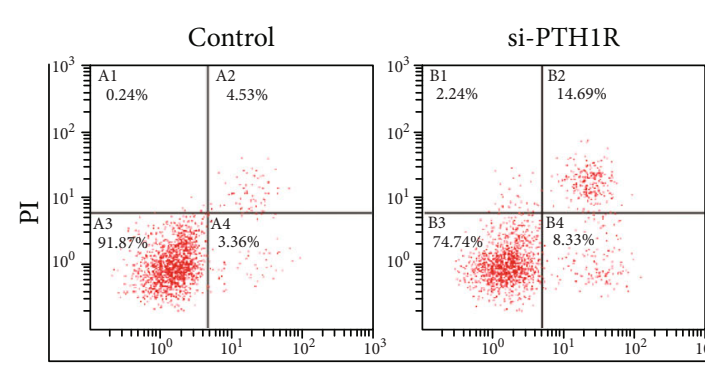

ALN-FOL-MTO-NLCs

ALN-FOL-MTO-NLCs
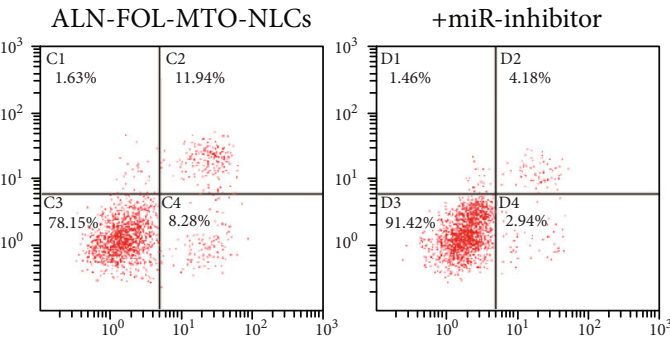

miR-mimics+pc-PTH1R

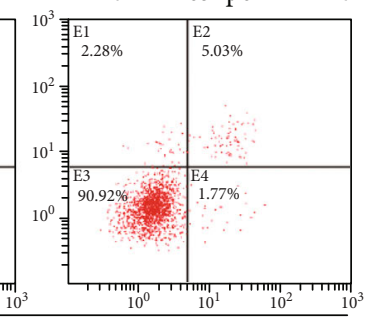

ANNEXIN V-FITC

(c)
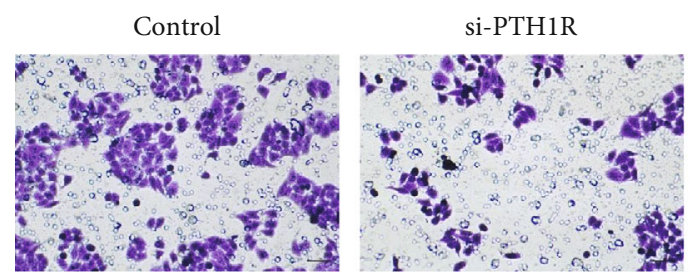

ALN-FOL-MTO-NLCs

ALN-FOL-MTO-NLCs +miR-inhibitor miR-mimics+pc-PTH1R
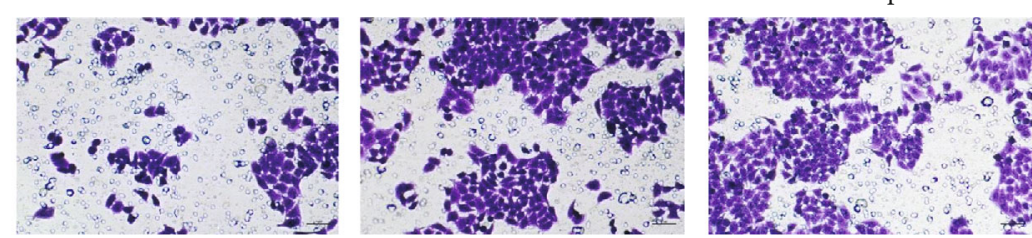

(d)

FIgURE 4: Reply experiment. (a) The relative expression level of PTH1R mRNA. (b) GCTB28 cell proliferation activity. (c) GCTB28 cell apoptosis rate. (d) GCTB28 cell invasion number. $(\bar{x} \pm s, n=3) . * * P<0.01$ compared with the pc-NC group; ${ }^{\#} P<0.05$ compared with the si-NC group; ${ }^{\triangle \triangle} \triangle P<0.001$ compared with the control group.

in shape, and the rate of drug coverage, particle size, and potential was consistent with the results of Shi et al. Furthermore, compared with MTO-NLCs, ALN-FOL-MTO-NLCs have a certain degree of improvement in terms of HA adsorption capacity, cellular uptake capacity, and toxicity to cancer cells. This improvement is partially attributed to ALN. As a 
bone metabolism regulator, ALN has a strong affinity with HA. Therefore, the modifications of nanoparticles with FOL not only ensured the steric hindrance of PEG but also increased the ingestion of nanoparticles by target cells. Immunofluorescence microscopy results showed that the ALN-FOR-MTO-NLC nanoparticles in GCTB28 cells were red fluorescent and mainly distributed in the cytoplasm, which is also consistent with the results of Vibet et al. [18].

Specifically, various studies have confirmed the expression changes of miR-125b in a variety of cancer tissues and cells. For example, miR-125b as a tumor suppressor gene was downregulated in breast cancer tissues and cells [19], and miR-125b expression in gastric cancer tissues and cells was also significantly downregulated [20]. In this study, it was found that the expression level of miR-125b in GCT tissues and cells was also significantly downregulated. The expression of miR-125b in GCT cells was upregulated to varying degrees after treatment with MTO-NLCs and ALN-FOL-MTO-NLCs, and the overexpression of miR-125b produced certain effects on proliferation, invasion, and apoptosis of GCT cells. Therefore, we think miR-125b played a role in the occurrence and development of GCT, which may be related to the role of ALN-FOLMTO-NLC nanoparticles.

In this study, inhibiting the expression of PTH1R in GCTB28 cells reduced the proliferation and invasion of GCTB28 cells and induced its apoptosis. Moreover, bioinformatics analysis showed that PTH1R and miR-125b have partially complementary sequences, and their targeting relationship was further verified via the dual-luciferase reporter gene experiment, Western blotting, and RTqPCR.

At the end of the study, through a series of rescue experiments, it was confirmed that ALN-FOL-MTO-NLCs inhibited the malignant behavior of GCTB28 cells by upregulating the expression level of miR-125b which then inhibited the expression of PTH1R. This result provides a theoretical basis for clarifying the mechanism of ALNFOL-MTO-NLCs in the treatment of GCT, and ALNFOL-MTO-NLCs combined with surgery may become a new strategy for the treatment of GCT.

\section{Data Availability}

All the data is available with the handwritten notebook documented in our lab.

\section{Conflicts of Interest}

The authors declare that there are no conflicts of interest regarding the publication of this paper.

\section{Acknowledgments}

This work supported by the Weifang City Health and Family Planning Commission Scientific Research Project Plan (grant no. wfwsjs-2018-107).

\section{Supplementary Materials}

Figure S1: GCTB28 cell apoptosis rate. Figure S2: GCTB28 cell invasion number. Figure S3: GCTB28 cell apoptosis rate. Figure S4: GCTB28 cell invasion number $(\bar{x} \pm s, n=3) ; * P$ $<0.05, * * P<0.01$, and $* * * P<0.001$, compared with the control group. (Supplementary Materials)

\section{References}

[1] B.-J. Noh and Y.-K. Park, "Giant cell tumor of bone: updated molecular pathogenesis and tumor biology," Human Pathology, vol. 81, pp. 1-8, 2018.

[2] E. Palmerini, P. Picci, P. Reichardt, and G. Downey, "Malignancy in giant cell tumor of bone: a review of the literature," Technology in Cancer Research \& Treatment, vol. 18, article 1533033819840000, 2019.

[3] J. Lüke, M. Hasenfratz, P. Möller, and T. F. E. Barth, "New aspects on giant cell tumor of bone," Der Pathologe, vol. 39, no. 2, pp. 125-131, 2018.

[4] H. Cheng, A. Chawla, Y. Yang et al., "Development of nanomaterials for bone-targeted drug delivery," Drug Discovery Today, vol. 22, no. 9, pp. 1336-1350, 2017.

[5] S. Aftab, A. Shah, A. Nadhman et al., "Nanomedicine: an effective tool in cancer therapy," International Journal of Pharmaceutics, vol. 540, no. 1-2, pp. 132-149, 2018.

[6] B. Bahrami, M. Hojjat-Farsangi, H. Mohammadi et al., "Nanoparticles and targeted drug delivery in cancer therapy," Immunology Letters, vol. 190, pp. 64-83, 2017.

[7] S. Qadri, Y. Haik, E. Mensah-Brown, G. Bashir, M. J. Fernandez-Cabezudo, and B. K. al-Ramadi, "Metallic nanoparticles to eradicate bacterial bone infection," Nanomedicine, vol. 13, no. 7, pp. 2241-2250, 2017.

[8] I. Takeuchi, S. Kobayashi, Y. Hida, and K. Makino, "Estradiol-loaded PLGA nanoparticles for improving low bone mineral density of cancellous bone caused by osteoporosis: application of enhanced charged nanoparticles with iontophoresis," Colloids and Surfaces B: Biointerfaces, vol. 155, pp. 35-40, 2017.

[9] S. Huang, X. Song, T. Li et al., "Pellet coculture of osteoarthritic chondrocytes and infrapatellar fat pad-derived mesenchymal stem cells with chitosan/hyaluronic acid nanoparticles promotes chondrogenic differentiation," Stem Cell Research \& Therapy, vol. 8, no. 1, p. 264, 2017.

[10] S. Jie, X. Guo, and Z. Ouyang, "Tumor ablation using novel photothermal Nax WO3 nanoparticles against breast cancer osteolytic bone metastasis," International Journal of Nanomedicine, vol. 14, pp. 7353-7362, 2019.

[11] J. Liu, Y. Zeng, S. Shi et al., "Design of polyaspartic acid peptide-poly(ethylene glycol)-poly(epsilon-caprolactone) nanoparticles as a carrier of hydrophobic drugs targeting cancer metastasized to bone," International Journal of Nanomedicine, vol. 12, pp. 3561-3575, 2017.

[12] Y. Shi, Z. Su, S. Li et al., "Multistep targeted nano drug delivery system aiming at leukemic stem cells and minimal residual disease," Molecular Pharmaceutics, vol. 10, no. 6, pp. 2479-2489, 2012.

[13] M. Pu, J. Chen, Z. Tao et al., "Regulatory network of miRNA on its target: coordination between transcriptional and posttranscriptional regulation of gene expression," Cellular and Molecular Life Sciences, vol. 76, no. 3, pp. 441-451, 2019. 
[14] S. Nishimori, F. Lai, M. Shiraishi et al., "PTHrP targets HDAC4 and HDAC5 to repress chondrocyte hypertrophy," JCI Insight, vol. 4, no. 5, 2019.

[15] R. Zhang, J. Li, G. Assaker et al., "Parathyroid hormone-related protein (PTHrP): an emerging target in cancer progression and metastasis," Advances in Experimental Medicine and Biology, vol. 1164, pp. 161-178, 2019.

[16] R. W. Cowan, G. Singh, and M. Ghert, "PTHrP increases RANKL expression by stromal cells from giant cell tumor of bone," Journal of Orthopaedic Research, vol. 30, no. 6, pp. 877-884, 2012.

[17] F. Wang, D. Yu, Z. Liu et al., "MiR-125b functions as a tumor suppressor and enhances chemosensitivity to cisplatin in osteosarcoma," Technology in Cancer Research \& Treatment, vol. 15, no. 6, pp. NP105-NP112, 2016.

[18] S. Vibet, K. Mahéo, J. Goré, P. Dubois, P. Bougnoux, and I. Chourpa, "Differential subcellular distribution of mitoxantrone in relation to chemosensitization in two human breast cancer cell lines," Drug Metabolism and Disposition: The Biological Fate of Chemicals, vol. 35, no. 5, pp. 822-828, 2007.

[19] G. Hu, X. Zhao, J. Wang et al., "miR-125b regulates the drugresistance of breast cancer cells to doxorubicin by targeting HAX-1," Oncology Letters, vol. 15, no. 2, pp. 1621-1629, 2018.

[20] X. Zhang, J. Yao, K. Guo et al., "The functional mechanism of miR-125b in gastric cancer and its effect on the chemosensitivity of cisplatin," Oncotarget, vol. 9, no. 2, pp. 2105-2119, 2018. 